SLAC-PUB-8096

March 1999

\title{
The Next Linear Collider Extraction Line Design ${ }^{1}$
}

\author{
Y. Nosochkov, T. O. Raubenheimer, K. Thompson and M. Woods \\ Stanford Linear Accelerator Center, Stanford University, Stanford, CA 94309
}

\begin{abstract}
The two main functions of the NLC extraction line include: 1) transmission of the outgoing disrupted beam and secondary particles to the dump with minimal losses; and 2) beam diagnostics and control. In this report, we describe the extraction line optics, present the results of tracking studies, and discuss the extraction line instrumentation.
\end{abstract}

Presented at the 1999 IEEE Particle Accelerator Conference (PAC99)

New York City, New York, March 29 - April 2, 1999

\footnotetext{
${ }^{1}$ Work supported by Department of Energy contract DE-AC03-76SF00515.
} 


\title{
THE NEXT LINEAR COLLIDER EXTRACTION LINE DESIGN ${ }^{\dagger}$
}

\author{
Y. Nosochkov $\ddagger$, T. O. Raubenheimer, K. Thompson and M. Woods \\ Stanford Linear Accelerator Center, Stanford University, Stanford, CA 94309
}

\begin{abstract}
The two main functions of the NLC extraction line include: 1) transmission of the outgoing disrupted beam and secondary particles to the dump with minimal losses; and 2) beam diagnostics and control. In this report, we describe the extraction line optics, present the results of tracking studies, and discuss the extraction line instrumentation.
\end{abstract}

\section{INTRODUCTION}

The power of the Next Linear Collider (NLC) [1] beams at $1 \mathrm{TeV}$ (cms energy) can be as high as $10 \mathrm{MW}$ and has to be safely disposed after the interaction point (IP). For the NLC beam parameters at the IP, a significant disruption [2] of the beam distribution occurs due to the beam-beam interaction, notably an increase in the beam angular divergence and energy spread. In addition, the beam collisions generate a significant amount of beamstrahlung photons, low energy $e^{+} e^{-}$pairs and other secondary particles; the number of the beamstrahlung photons from the IP is comparable to that of the primary beam particles. The need to transport the photon power to the dump places constraints on the extraction line design. Following earlier studies [3], the current design is based on a shared dump for the primary leptons and photons. To minimize beam losses due to large energy spread in the disrupted beam, it is critical to design optics with large chromatic bandwidth. Additional constraints are imposed by planned diagnostics after IP.

Several scenarios are currently under study for NLC beam parameters [1]. In this paper, we present the results for one scenario which gives the largest energy spread and beam loss for the disrupted beam. This set of beam parameters includes: $1046 \mathrm{GeV}$ cms energy, $120 \mathrm{~Hz}$ repetition rate, 95 bunches per RF pulse and $0.75 \cdot 10^{10}$ bunch charge (see also Table 1). Since the colliding beam parameters are identical at IP, the extraction line design described below is applicable to both beams.

\section{LATTICE}

The primary requirement for the NLC extraction line is to transport the outgoing beams to the dump with minimal losses and provide conditions for beam diagnostics. The main optics includes: 1) a set of quadrupoles after IP to focus the outgoing lepton beam; 2) a horizontal chicane and secondary IP in the middle of chicane for beam measurements; and 3) a second quadrupole set at the end of the line to make a parallel beam at the dump. The strength of

\footnotetext{
$\dagger$ Work supported by the Department of Energy Contract DE-AC03$76 \mathrm{SF} 00515$

‡E-mail: yuri@slac.stanford.edu.
}

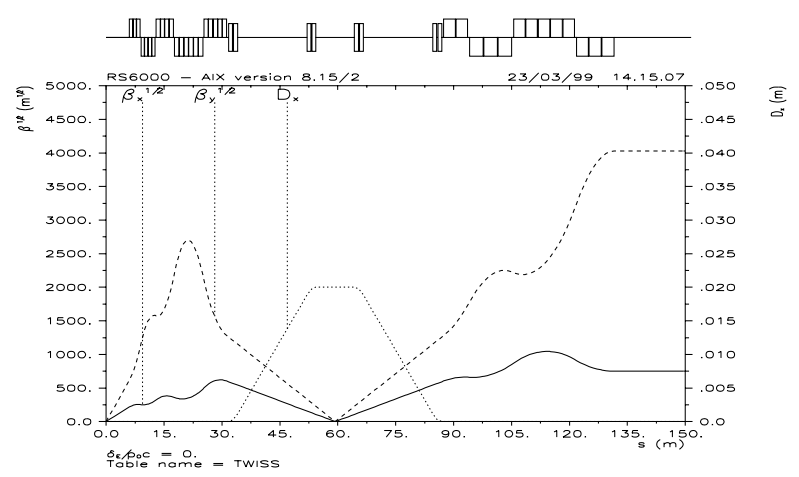

Figure 1: Lattice functions in the extraction line.

the first quadrupole system is defined by the point-to-point transformation from IP to secondary IP $\left(R_{12}=R_{34}=0\right)$, and the second set of quadrupoles provides a point-to-parallel transformation to the dump $\left(R_{22}=R_{44}=0\right)$. The optics calculations were made using MAD code [4].

The lattice functions of the extraction line are shown in Fig. 1. Since the beam size at IP is much smaller in the vertical plane, the first quadrupole after IP focuses the beam horizontally. This minimizes the overall beam size in the extraction line. For realistic magnet design, we use the quadrupole pole tip field $\leq 12 \mathrm{kG}$ at $1 \mathrm{TeV}$ (cms energy) and $8.2 \mathrm{kG}$ field in the bends. The total length of the beam line is about $150 \mathrm{~m}$.

The beam line optics is constrained by the following parameters and requirements:

- Crossing angle and positions of the final focus quads,

- Disrupted beam parameters at IP,

- Angular divergence of the beamstrahlung photons,

- Shared dump for a primary beam and photons,

- Secondary IP and chicane for beam diagnostics.

\subsection{IP Constraints}

The NLC beams cross at $20 \mathrm{mrad}$ horizontal angle, and the nearest final focus quadrupoles are placed $2 \mathrm{~m}$ before the IP. To minimize geometric interference between the final focus and extraction line magnets, the latter should be placed as far as possible from the IP. However, a long free space after IP increases the beam size, apertures and length of the extraction line quadrupoles. In this design, we place the first extraction line quadrupole $6 \mathrm{~m}$ after IP. This clears the first three final focus quadrupoles.

\subsection{Disrupted Beam Parameters}

The strong beam-beam interactions change the beam parameters at IP. Notably, a significant increase occurs in the beam energy spread and horizontal angular divergence. The horizontal phase space for the disrupted primary beam 


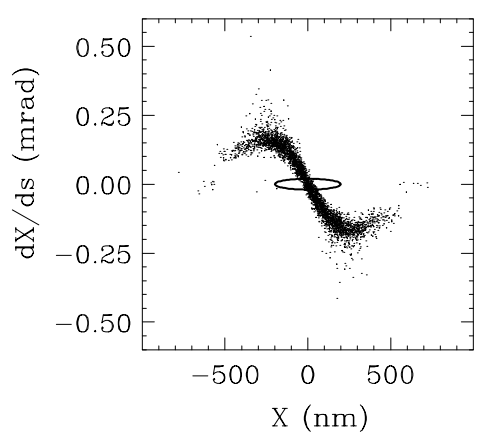

Figure 2: Horizontal phase space at IP: dots - disrupted beam, ellipse - $1 \sigma$ undisrupted beam.

Table 1: Beam parameters at IP.

\begin{tabular}{lcc}
\hline Beam parameter & Undisrupted & Disrupted \\
& $\boldsymbol{x} / \boldsymbol{y}$ & $\boldsymbol{x} / \boldsymbol{y}$ \\
\hline Emit. $(\mathrm{m} \cdot \mathrm{rad})\left[10^{-13}\right]$ & $39 / 0.59$ & $120 / 1.02$ \\
rms size $(\mathrm{nm})$ & $198 / 2.7$ & $198 / 3.2$ \\
rms divergence $(\mu \mathrm{rad})$ & $20 / 22$ & $125 / 33$ \\
$\beta^{*}(\mathrm{~mm})$ & $10.0 / 0.125$ & $3.259 / 0.103$ \\
$\alpha^{*}$ & $0 / 0$ & $1.805 / 0.306$ \\
\hline
\end{tabular}

at IP is shown in Fig. 2. The beam distribution was calculated using GUINEA PIG beam-beam simulation code [5]. The undisrupted $1 \sigma$ phase ellipse is shown for comparison in Fig. 2. The nominal and disrupted beam parameters are given in Table 1.

The energy distribution for the disrupted beam is shown in Fig. 3. The low energy tail extends to $\delta \sim-90 \%$ $(\delta=\Delta p / p)$, and up to $1 \%$ of the beam power $(\sim 100 \mathrm{~kW})$ is carried by the particles with $\delta<-50 \%$. To minimize losses in this energy range, the optics requires a huge chromatic bandwidth and large magnet apertures. The methods used to improve the chromatic transmission are discussed below.

\subsection{Chromatic Bandwidth}

To satisfy optics conditions for the nominal energy, the use of quadrupole doublets in the beginning and end of the extraction line is sufficient. However, the strong doublets significantly overfocus the particles in the low energy range and lead to beam losses. To reduce the overfocusing, we replaced the doublets by 5 alternating gradient quadrupoles in the beginning and 4 quadrupoles at the end of the beam line (see Fig. 1). Since the net focusing for the nominal energy has to remain the same, the strengths of individual quadrupoles are reduced. As a result, the low energy particles experience less focusing in each quadrupole and oscillate through the alternating gradient system with less overfocusing. The strengths of individual quads were optimized by minimizing the low energy betatron amplitudes. The limitations of the described multi-quad system are the increased length of the focusing system and the large beam size and magnet apertures.

A simplified explanation of the multi-quad bandwidth can be made using analogy with a FODO system of $n$ identical cells with fixed total phase advance $\mu$. In such a sys-

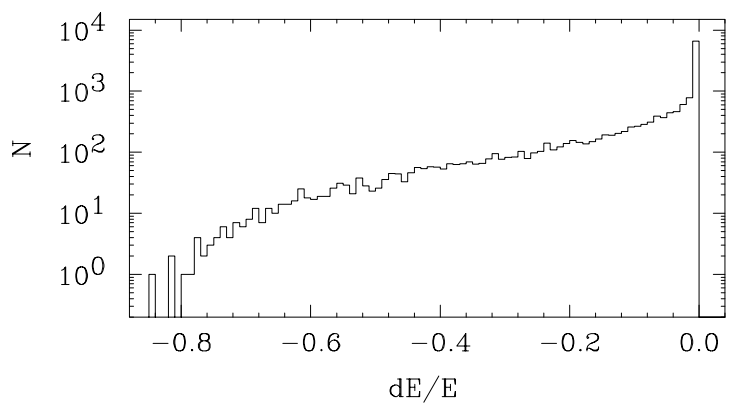

Figure 3: Energy distribution for disrupted beam.

tem, the range of low energies satisfying stability conditions increases with $n$ proportional to $1-\sin (\mu / 2 n)$.

\subsection{Beamstrahlung Photons}

Bending of the particle orbits due to beam-beam forces at IP results in radiation and significant flux of beamstrahlung photons from IP. The GUINEA PIG simulation shows that the rms angular spread of the photons is on the order of $\pm 100 \mu \mathrm{rad}$ in the horizontal plane and a factor of 2 smaller in the vertical plane. In this design, the primary beam and the photons are transported to one shared dump. For beam diagnostics in the extraction line, it is desirable to avoid any material in the path of the beamstrahlung photons. Therefore, large apertures of the magnets and beam pipe have to be used to include the photon flux. For this design, we assumed the maximum photon beam divergence of $\pm 1 \mathrm{mrad}$ and $\pm 0.5 \mathrm{mrad}$ in the horizontal and vertical planes, respectively.

\subsection{Chicane}

The horizontal chicane allows to separate the outgoing electron and photon beams for measurements. In this design, the chicane is made of 4 pairs of bends which produce a closed bump with $2 \mathrm{~cm}$ of horizontal displacement and dispersion. This dispersion is sufficient to measure the energy spread of the undisrupted beam at the secondary IP. Since there are no quadrupoles between bends, the orbit bump is closed for all energies. The maximum displacement for the low energy particles increases with $1 / E$.

\subsection{Magnet Apertures}

Large physical aperture is required for maximum transmission of the beam to the dump. We determined the extraction line aperture by: 1) $10 \sigma$ beam size; 2) low energy horizontal excursions in the chicane; and 3) the maximum photon flux size. Schematically, this is shown in Fig. 4. Outside the chicane region the aperture is dominated by the $\pm 1 \mathrm{mrad}$ horizontal angle of the photon flux. The quadrupole apertures vary from $\pm 1 \mathrm{~cm}$ for the first quadrupole after IP to $\pm 13 \mathrm{~cm}$ near the dump.

To minimize beam losses in the chicane region, we increased apertures to include low energy orbits up to $\delta=$ $-90 \%$. With the beam size included, the maximum horizontal aperture in the chicane is $\pm 20 \mathrm{~cm}$. Since the orbit excursions in the chicane occur in the horizontal plane, 


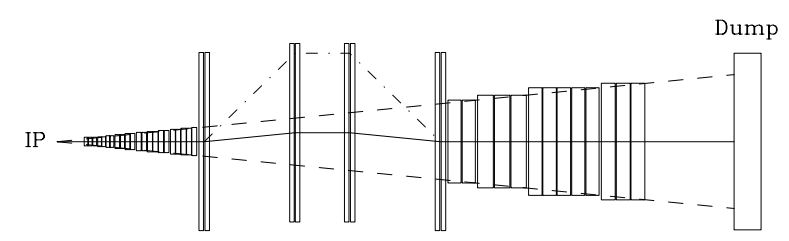

Figure 4: Aperture constraints: solid line - nominal beam; dash: \pm 1 mrad photon $x$-angle; dash-dot: $\delta=-90 \% x$-orbit.

a smaller vertical aperture can be used in the bends. The tracking simulations showed that $\pm 50 \mathrm{~mm}$ vertical gap in the bends is sufficient to minimize beam losses and include the $\pm 0.5 \mathrm{mrad}$ vertical photon angle.

\section{DIAGNOSTICS}

Beam line diagnostics fall into three categories: 1) standard diagnostics (BPMs, toroids and ion chambers) to facilitate cleanly transporting the beam to the beam dump; 2) luminosity diagnostics to measure and optimize the luminosity; and 3) physics diagnostics to measure the beam polarization, energy, and energy spread.

The luminosity diagnostics will include BPMs with 1 $\mu \mathrm{m}$ resolution for measurements of deflection angles, as well as detectors to monitor low energy particles produced at the IP from radiative Bhabha and pair production processes. The physics diagnostics will include a Compton polarimeter, an energy spectrometer, and a wire scanner to measure energy spread. The Compton polarimeter will collide a laser beam with the electron beam in the middle of the chicane, and its detector will analyze Comptonscattered electrons below $50 \mathrm{GeV}$ after the chicane. A conventional wire scanner in the chicane can be used for the energy spread measurements. An SLC-style energy spectrometer is planned between the chicane and the beam dump to measure the separation of synchrotron light due to a precisely calibrated spectrometer magnet.

\section{BEAM LOSS}

The methods used to improve the beam transmission included: 1) the use of multi-quad focusing systems for large chromatic bandwidth; and 2) sufficiently large magnet apertures. To assure accuracy of the beam transport with low energy tail, we used a modified version of the DIMAD code [6] which can handle chromatic terms to all orders.

With up to $10 \mathrm{MW}$ of the NLC beam power, even the loss of just $0.3 \%$ would be equivalent of losing the whole SLC beam $(30 \mathrm{~kW})$. Therefore, our goal was to reduce to a minimum the overall beam losses. In addition, an excessive beam loss would interfere with the planned diagnostics and experiments in the extraction line.

In tracking simulations, we used a disrupted distribution of 15,000 primary beam particles calculated with GUINEA PIG code. This distribution was tracked from IP to the dump and the beam losses were monitored along the beam

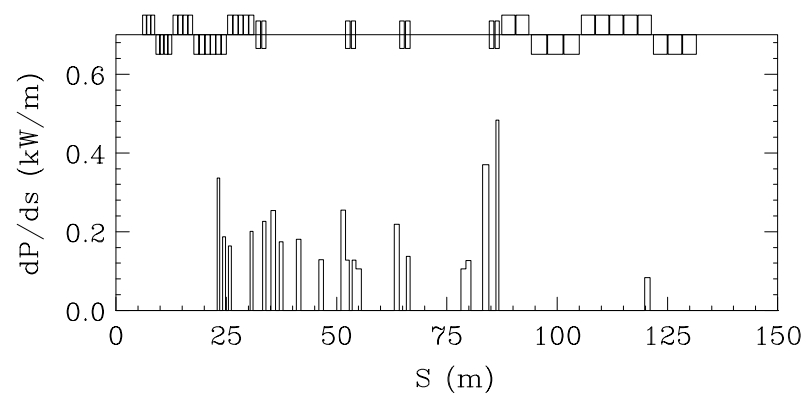

Figure 5: Distribution of the beam power loss.

line. We used round apertures for the quadrupoles and drifts, and rectangular aperture for the bends.

The most losses occur for the very low energy particles which experience strong overfocusing in quadrupoles and large horizontal deflections in the bends. The calculated beam power loss along the beam line is shown in Fig. 5. In this case, all particles with $\delta>-50 \%$ and most with the lower energies are transported to the dump. The total loss is $4.7 \mathrm{~kW}(0.25 \%$ particles $)$ and the distributed power loss is below $0.5 \mathrm{~kW} / \mathrm{m}$. At the dump, the rms $(x / y)$ beam size is $7.7 / 4.7 \mathrm{~mm}$ with the tails extending to $\pm 100 / 40 \mathrm{~mm}$.

The simulations included the $6 \mathrm{~T}$ detector solenoid (12 Tm after IP). Due to the crossing angle, the solenoid induces vertical orbit distortions. The study showed that with corrected vertical orbit after IP, the solenoid effect on the beam loss is negligible.

\section{FUTURE STUDIES}

In future studies, we need to increase statistics of GUINEA PIG simulations to obtain more accurately the distributions of electrons in the low energy tail and beamstrahlung photons at large angles. Tracking of these particles is needed to calculate signal to background ratios in the diagnostic detectors.

More details have to be included in the design of the beam line diagnostics, in particular the magnets for the energy spectrometer.

Methods of directing the main beam and photons to separate dumps and possibly reducing the backgrounds and neutron back-shine from the dump need to be investigated.

\section{REFERENCES}

[1] NLC ZDR Design Group, "Zeroth-Order Design Report for the Next Linear Collider," SLAC Report 474 (1996); with updated parameters at: http://www-project.slac.stanford.edu/lc/nlc-tech.html.

[2] R. Hollebeek, "Disruption Limits for Linear Colliders," NIM, 184, p. 333 (1981).

[3] J. Spencer, et al., "The SLAC NLC Extraction \& Diagnostic Line," Proceedings of the 1995 IEEE Part. Acc. Conf., Dallas, p. 713 (1995).

[4] H. Grote, F. C. Iselin, CERN/SL/90-13 (AP) Rev.4 (1994).

[5] D. Schulte, Ph.D. thesis (1996).

[6] P. Tenenbaum, et al., "Use of Simulation Programs for the Modelling of the NLC," these proceedings (1999). 